\title{
Research and Design of Hongqiao Airport Water Management Information System
}

\author{
Lanying Zhang \\ Business School, University of Shanghai for Science and Technology \\ Shanghai 200093, China \\ E-mail: lanyingzhang06@163.com \\ Chongjun Fan (Corresponding author) \\ Business School, University of Shanghai for Science and Technology \\ Shanghai 200093, China \\ Tel: 86-13801715020 E-mail: cjfan@sh163.net
}

Xiaodong Zhu

Business School, University of Shanghai for Science and Technology

Shanghai 200093, China

Jianzheng Yang

Business School, University of Shanghai for Science and Technology

Shanghai 200093, China

Wenchun Shu

Shanghai Airport Construction Headquarters, Shanghai 201202, China

Chen Zeng

Shanghai Airport Construction Headquarters, Shanghai 201202, China

The research is financed by the research project of Science and Technology Commission of Shanghai Municipality of China. No. 062112051, the key research project of Shanghai Municipal Education Commission of China. No. 06ZZ34, and Shanghai Development of Undergraduate Education Base III-Electronic Commerce (Sponsoring information)

\begin{abstract}
According to the general planning requirements of Outside Management Center (OMC for short) of Hongqiao Airport and on the basis of water management business description, this paper divides three levels for the water information system management structure, designs the overall structure of the water information system, analyses the function of the system structure, and then applies the data mining technology in the layer of water management decision, finally gives data levels of data mining system. This project can provide reference and help to related Chinese airport's planning and construction projects.
\end{abstract}

Keywords: Airport construction, Information system, Water management, Data mining, E-Business

\section{Introduction}

With the world economic growth center transferring to Asia-Pacific region and China becoming the new economic growth point of the world, the State Civil Aviation proposed in the 2010 strategic planning that we will build Beijing, Shanghai and Guangzhou airports to be the international hub airports and drive them to participate in the competition among other international hub airports. According to the strategic planning, Hongqiao airport 
will meet 30 million passengers per year, and it will develop to be a complex hub airport. With the increasing construction scale of Hongqiao airport, the operation and management of the airport need more coordination, the operation security becomes more and more important, and the traditional management methods of the airport nearly cannot meet the growing demand for efficient management, so these problems promote the major hub airport in the world centralized coordination management to enhance the efficiency, security operation and improve the response and the abilities of handling emergency cases.

According to the regional principles of Hongqiao airport business management, it can be divided into three parts-the flight area, terminal area and outside area. The airport operation center is mainly in the charge of the information management in flight area; terminal operation center is in the charge of terminal area; the outside management center is in the charge of facilities outside the airport and it provides a set of intelligent electro-mechanical system aiming at safely supplying its services, which is also in charge of the utility systems including water supply, power supply and distribution, energy and roads, and helps the production, equipment maintenance, customer services and so on. This paper emphasizes building an information platform for the OMC water management system to change the original management which is disperse and manual-based operation, and it builds up a foundation for Hongqiao airport OMC water management, command schedule and monitor.

The information management system mentioned in this paper which is based on OMC water systems of the outside facilities in the Hongqiao airport has a great significance. On the one hand, there are few research literatures on water information system, so the literatures can be referred are few. Only some literatures study the water information, while there are few literatures related the design of water management information system. On the other hand, Hongqiao airport $\mathrm{OMC}$ water system is a large-scale engineering project. And this paper suggests that the data mining techniques is applied to the system to help the senior managers make decision. Therefore, the research in this paper has great significance to the optimization of water resources for other similar domestic airport projects.

\section{Water Management Information System}

Water management information system is designed to provide business support for Hongqiao Airport OMC and technical support for centralized management, which is on the basis of water monitoring, by using the computer technology and communication technology, achieve water information collection, transmission, storage, maintenance and analysis.

\subsection{Business description of water management system}

Water management center is mainly responsible for monitoring and management of sewage disposal, operation, management and maintenance of feed pump and drainage pump, monitoring and management of a variety of equipment and facilities which are involved in the work.

OMC water Scheduling layer needs these main functions: workspace south pumping station(the water supply pumping station), monitoring and controlling the operational status of water supply pumping station; south and north pumping station(including regulation pools)in flight area, monitoring and controlling the operational status of drainage pumping station; workspace south and north pumping station(the part of rain and sewage), monitoring and controlling the operational status of drainage pumping station; monitoring water supply pipe network, mainly within the scope of Hongqiao airport west district; reserved the reclaimed water system whose information will be included in the water monitoring system. It can be seen as a node of information system and accessed to OMC system when the reclaimed water system is constructed in future.

\subsection{Water management-level division}

Water management-level is designed for three levels: decision support layer, business layer and on-site implementation layer. Business layer is the core.

Layer 1: decision support layer--or center information layer, mainly serve OMC general duty and top-level managers which control the water performance, harmonize and manage when something goes wrong. Analyst can inquire the water level, analyse the important data, make strategy, plan business and so on.

Layer 2: business operations layer - or water scheduling layer, mainly serve user departments to carry out their daily work. Besides monitoring operation of per pumping station, the system also monitor the water supply pipe network within the jurisdiction of OMC.

Layer 3: on-the-spot implementation layer, mainly serve the scene of equipment. On-the-spot the staff maintain and manage these equipments which include workspace south and north pumping station, south and north regulation pools, workspace south water supply pumping station. 


\subsection{The main technologies and applications of dynamic monitoring water equipment}

\subsubsection{Supervisory Control and Data Acquisition — or SCADA for short}

SCADA system is one of the four remote technologies (telemetry, remote control, remote signalling and remote regulating) of computer systems. It uses many technologies such as computer, communications, control, display, etc, which is used in various water supply and drainage systems to achieve the real-time data collection, transmission, processing and other work. When necessary it can achieve automatic acquisition and processing remote information for some elements of the site and control these information aiming at protecting optimal scheduling of water supply and drainage systems for airport.

The application of SCADA in the OMC water system has two meanings. The first is distributed data acquisition system in the on-the-spot layer. That is the lower computer system. The second is data processing and display system in the scheduling layer. That is the upper computer system.

SCADA system performs some specific functions. Data acquisition and information sent control which use the data collection method based on inter-process communication and commonly use the service program of a variety of intelligent data acquisition equipment; alarm processing and event handling which will issue sound and light alarm and display the content in the alarm window when there are abnormal phenomenon. Acoustic alarm can be eliminated after confirmation and also be eliminated at certain times. Recording the alerting content on the list of database events, if it needed, you can call up it on request and print it as ordered. These alarms that had been identified can be put into alarm databases with timestamp. These alarms that had not been identified should continue to issue sound and light alarm until the staff on duty confirm; Displaying and recording the historical trend which provide data records, query and graph display of monitoring point based on the special real-time database and simultaneously provide quite helpful functions in the industrial process control and management for the need of control and management; the function of network communication which provides the non-transparent network communication mechanism can build distributed monitoring and processing power of the upper computer; the open system function which provides the connection with other applications to exchange data and other database systems.

\subsubsection{Programable Logic Controller-or PLC for short}

PLC can achieve semi-unattended or unattended by setting the running program which monitors the entire pump station.

PLC system performs some specific functions. Take the case of workspace south pumping station (the water supply pumping station) as example. Remote or on-the-spot monitoring and control the opening and closing water pumps; monitoring the value of inlet pressure, outlet pressure and backwater pressure, recording the value of real-time monitoring and displaying alarm when there is an failure; monitoring the value of the flow which include the flow of inlet and the flow of outlet, recording the value of real-time monitoring and displaying alarm when there is an failure; monitoring the value of the turbidity which include the turbidity of inlet and the turbidity of outlet, recording the value of real-time monitoring and displaying alarm when there is an failure; monitoring the value of the free chlorine which include the free chlorine of inlet and the free chlorine of outlet, automatically adjusting the amount of increased chlorine according the amount of the free chlorine of inlet, recording the value of real-time monitoring and displaying alarm when there is an failure; monitoring the value of the $\mathrm{PH}$ which include the $\mathrm{PH}$ of inlet and the $\mathrm{PH}$ of outlet, recording the value of real-time monitoring and displaying alarm when there is an failure; monitoring the automatic chlorination equipment, automatically adjusting the amount of increased chlorine according the amount of the free chlorine of inlet, and displaying alarm when there is an failure; monitoring the liquid level of clean-water reservoir, recording the value of real-time monitoring and displaying alarm when there is an failure.

\subsubsection{Configuration Software--Intouch}

The upper computers with the configuration software-Intouch are connected to the PLC of the pumping stations by an Ethernet Switch. Through the upper computer system, equipment operation record and alarm record data which are collected by the PLC of the pumping stations will be automatically included into the database in order to be queried conveniently; On the other hand, in order to generate reports to be queried conveniently, the upper computer system will include the running time of the water pump and patrol records into the database.

\subsection{The design of water system structure}

The water system consists of Decision Support Information Center, Central Control Room, PLC, and Communication Network. The SCADA system which includes Decision Support Information Center, Central 
Control Room, and PLC will achieve centralized management and control of a variety of equipment operation in the whole airport through data collection, transmission and exchange. The overall structure of the system is shown in Figure 1.

\subsection{The applications of water system}

\subsubsection{Monitoring the pumping station equipments}

According to various information which is generated in the process of the pumping operation, we test and evaluate the cause, and then decide which measures to maintain and prevent the equipment. Monitoring equipment includes Signal Acquisition and Processing which include sensors converting the information into electrical signals and programs of signal processing. In this system, the Signal Generator consists of several sensors. These are liquid-level sensor, flow Sensor, main loop current Sensor, temperature sensor, the switch of pump valve, etc.

\subsubsection{Signal Acquisition and Processing}

The structure of signal acquisition and processing is shown in Figure 2.

The lower computer of the pump station (or secondary instrument) and the configuration software-Intouch can control the process of signal acquisition and processing through PLC.

The dynamic data of equipment operation can be acquired by the configuration software-Intouch.

\section{Data mining of decision support layer}

\subsection{Demand analysis of data mining}

Data mining is the process of extracting implicit, unknown and potentially useful knowledge from vast, incomplete, noisy, vague and random data by using scientific methods in fields of mathematics, statistics, artificial intelligence and machine learning.

The following is a brief explanation about the relationship among data mining, data warehousing and online analytical processing in water systems business intelligence of airport management center OMC. From the perspective of data mining, data mining has a high requirement for data environments. In the process of data mining, after a clear understanding of problems to be resolved, an important task is to select the target data set, namely, pre-processing the source data, obtaining high-quality data for mining and establishing a good data environment. This work is an extremely difficult and time-consuming step in the data mining process, and is the foundation and key to ensure the effective and correct implementation of data mining as well. Data warehouse technology can greatly meet the demand of data mining technology for the data environments. Data warehouse is subject-oriented, integrated, relatively stable, and time-varying data sets for better support policy-makers of businesses and organizations to analyze and process. Although the data warehouse is not a necessary condition for data mining, the characteristics of the data warehouse makes data mining more effective. From the perspective of a data warehouse, data mining is the advanced stage and supplement of online analytical processing OLAP. Moreover it can provide more in-depth, detailed, effective and potential decision-making knowledge to give more effective support to decision-makers on the basis of OLAP.

For Hongqiao Airport OMC water supervisor, the large and complex data report and monitoring information of water running are important and essential components to ensure the normal operation of airports. Water systems layout so many monitoring sites that the intelligent devices produce such large amounts of data in real-time operation. Meanwhile each monitoring site observes year after year, which also produce mass of data collected and accumulated. It generally is difficult for people to understand these data and their relationships, not to mention timely summary and forecast. We could learn about equipment performance and operational status from these data, automatically extract the knowledge or rules from the data generated in real time by the computer, and use the knowledge gained to forecast and estimate the upcoming course or timely correct the process of deviation occurred according to the knowledge, then reasonably arrange scheduling programs and improve operational efficiency.

\subsection{Technical Data-hierarchy of data mining}

Water management data mining system is deepened on the platform of the water management information system. Water data analyst should be equipped. Integration of data mining techniques and tools, by constructing a data warehouse, online analytical processing OLAP and data mining, we could discover hidden potential and valuable knowledge or rules from a large number of water data reports and monitoring information. Then OMC water supervisor and the airport leaders make decisions with these knowledge and rules. Figure 3 shows data hierarchy of the OMC water data mining system. 
The arrows denote the process in Figure 3. Key data include water system data, data warehouse, predictive models and metadata. Data layer is divided into three layers, namely, data acquisition layer, data storage layer, the data show layer, and each layer contains the data as the figure shows. We divide the realizable process of OMC water system data mining into the data source collection, ETL process, OMC water systems data warehouse, OLAP, data mining, predictive models and meta data management and other key technologies and processes.

\subsection{Complex data analysis methods}

In view of water data analysis, we discuss some complex data analysis and prediction methods in application of water data mining system.

We discuss the neural network method used in analysis of water nonlinear data. For analysis and prediction of water, first we can use seasonal data to extract periodic law, then use neural network model for nonlinear modeling.

Compared to the traditional statistics forecasting method, for the nonlinear prediction problem, forecasting precision of the neural network method is improved, and because this method has the characteristics of parameters, the modeling process does not require deep knowledge of the subject, and when it is applied in the prediction field, the whole process can be realized automatically on the computer, it's loved by the data processing personnel.

We also studied how to use experience and relate data comprehensively by using the Bayes to improve efficiency of data analysis.

Bayes time series analysis and prediction method is a system modeling method developed in recent decades. The basic idea is that combining people's experience of knowledge with practical model as priori information knowledge, namely the comprehensive utilization of the model information, data information and priory information for analysis and prediction.

Take the airport using water energy as example, compared to natural science systems, it often has a rapidly changing. In a condition of no sufficient observation data, the mathematical model has changed, so it is difficult for mathematical modeling. By the ideas of Bayes, using the relevant data to improve mathematical modeling ability is a valuable approach to be discussed.

\subsection{Decision Support example of application Data Mining}

Data mining technology in water management information systems of OMC has the potential application values. For example, the dropsy and drainage on runway in rainy and snowy day must be below the grounding of lights in order to ensure lamp navigation function, and at the same time the water level of underpass must maintain a certain water balance to prevent the lower ground from collapsing.

In the water management system, by using data mining technology, we can find the hidden relationship between water level and weather from the collected data of the water, as well as the relationship between the water level and year, season, month. Whereby the possible changes of water level in the future can be predicted which lay a foundation for more accurate water management, rational use of water resources, and the infrastructure security.

Meanwhile water management system needs the data mining technology to do data statistical analysis of the monthly water consumption, sewage treatment water, pumping stations discharge to the drainage, maintenance of equipment condition of various user units in the airport in order to learn the water use in the airport. After data analysts analyze the data, related water-energy-saving options could be developed for resource optimization.

\section{Developing E-business}

In the above parts, the point of water management information system construction in airport management center is about the information management for the running of water and analyzing the data of water to achieve water resources economy. In future, taking into consideration, there are many single buildings and different users in Hongqiao airport. For the management of water resource usage and the state of pay cost, it is needed for the executive to achieve the communion with users. So we plan to establish the E-business System based on the water management information system and lay a good basis for management center of water to continually satisfy requirement of users.

The emergence of e-commerce needs data exchanged independently and freely, and also needs computerize transaction between the manager and users. The system of e-commerce can achieve online exchange and direct payment. Developing e-business enables the water management information system to achieve online exchange through the local area network. 


\section{Conclusions}

Extension project of Hongqiao airport is a complex system engineering. OMC is on basis of general airport Business. The previous labor control and Integration Management in this extension project require new management mode to resolve wider scale management problems.

This study is on the basis of the previous water running conditions and extension project of Hongqiao Airport, for reference of water management information system designing. The study of water management information system, especially date mining in the field looks forward to further research. For instant, water running statistical analysis, the relationship between water usage and season or weather and the relationship between water usage and volume of commuters or flights.

OMC system building of Hongqiao Airport is on top level in China. The study and conclusion on water management information system will inspire later airport construct.

\section{Acknowledgements}

This research was supported by the research project of Science and Technology Commission of Shanghai Municipality of China (No. 062112051), the key research project of Shanghai Municipal Education Commission of China (No. 06ZZ34), and Shanghai development of Undergraduate Education Base III-—Electronic Commerce. Express the thanks.

\section{References}

Dong xiaohui, Zhang xiuyu. (2006). Analyze the construction of data warehouse feature-based water conservancy decision supporting system. Automation in water resources and hydrology, 1: 23-25.

Fan chongjun, Xiong honglin, Zhang haiying, Liu wujun. (2009). Analysis of network security and risks prevention strategies of Hongqiao Airport West Terminal, Computer and Information Science, 2(3): 46-52.

Fan chongjun. (2009). Measure nonlinear dependence between time series based on correlation dimension, Journal of Systems Science and Information, 7(1): 1-9.

Fan chongjun. (2009). One kind of Nonlinear Model Approach with Neural Networks and Its Application in forecasting, Journal of Information and Decision Science, 4(2): 105-113.

$\mathrm{Gu}$ chengdong, Liu wujun. (2007). Management and planning of transport hub construction of Shanghai Hongqiao, Comprehensive Transportation, 9: 54-57.

Jiawei Han, Micheline Kamber. (2008). Data Mining Concepts and Techniques, Beijing: China Machine Press.

Luo fengman, Zhou xinzhi, Gu zhongbi. (2005). Structure design of water resources planning intelligent decision support system, Yellow river, 27(8): 30-31.

Wu Nian zu. (1999). The Construction of Pudong International Airport-Information System. Shanghai: Publishing House of Shanghai Science and Technology.

Wu Nian zu. (2008). The construction of operational information system of Pudong International Airport. Shanghai: Publishing House of Shanghai Science and Technology. 


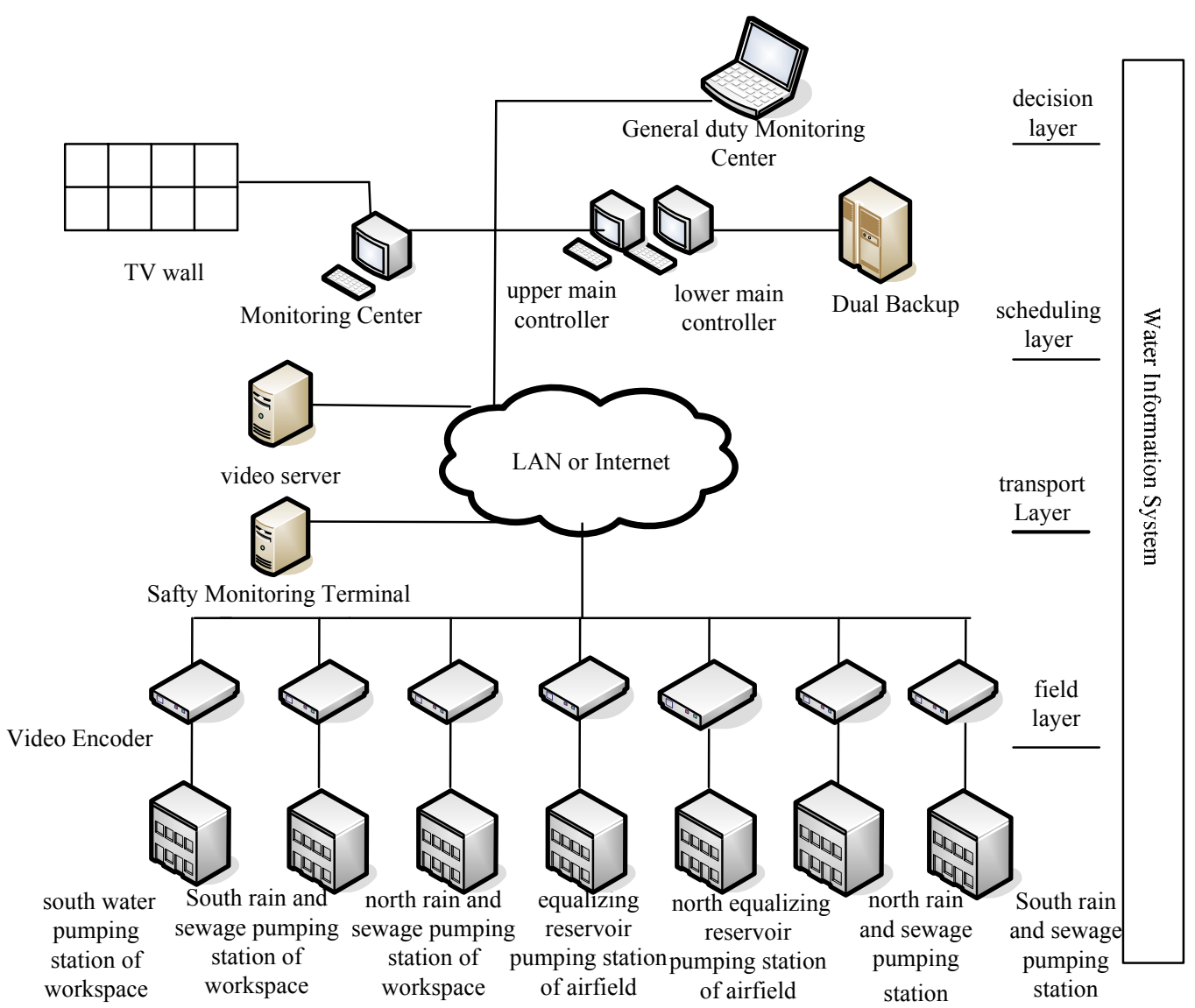

Figure 1. The overall structure of Water Management Information System

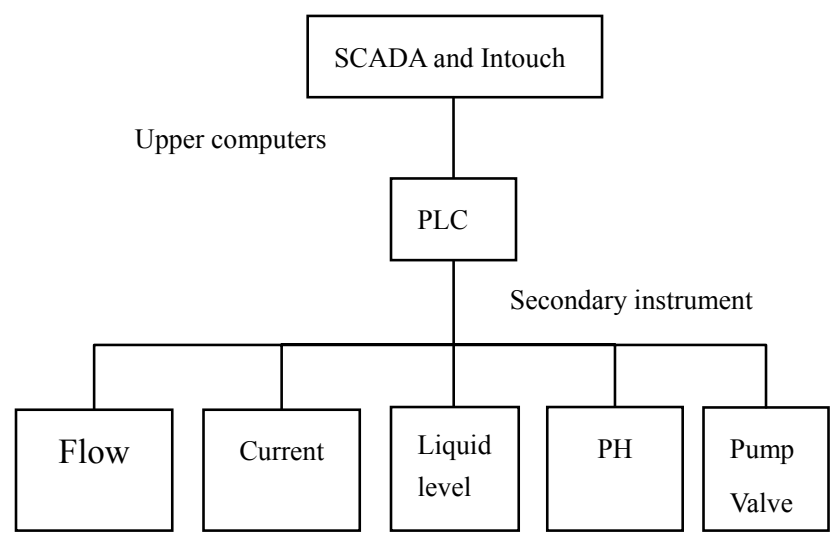

Figure 2. The structure of signal acquisition and processing 


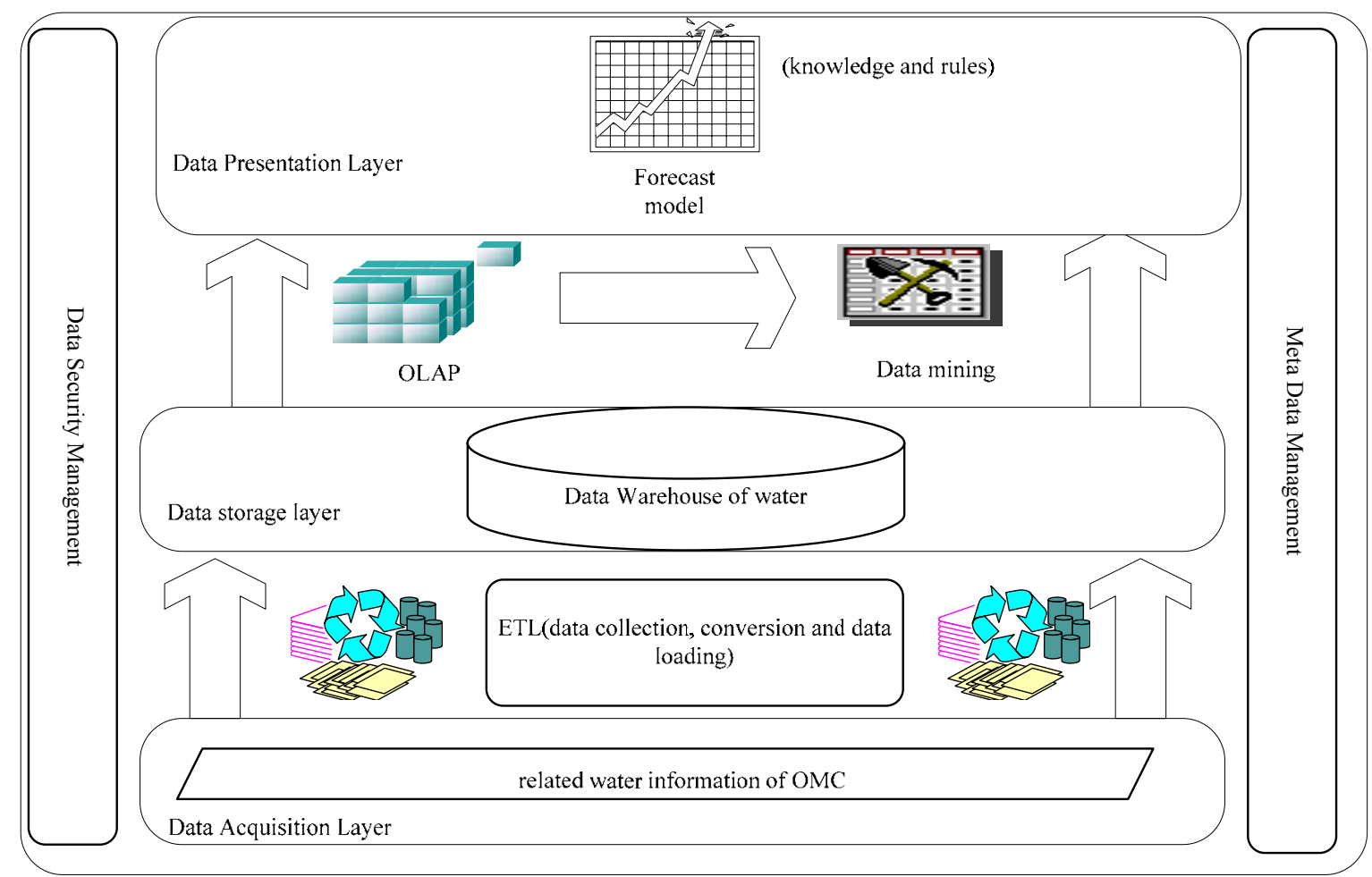

Figure 3. The data hierarchy of the OMC water data mining system 